\title{
Structural Distortions in the Paramagnetic Insulating Phase of $\mathrm{La}_{0.7} \mathrm{Ca}_{0.3} \mathrm{MnO}_{3}$
}

\author{
C.S. Nelson, ${ }^{1}$ Y.J. Kim, ${ }^{1}$ J.P. Hill, ${ }^{1}$ Doon Gibbs, ${ }^{1}$ V. Kiryukhin, ${ }^{2}$ T.Y. Koo, ${ }^{2,3}$ and S-W.
} Cheong,

${ }^{1}$ Department of Physics, Brookhaven National Laboratory, Upton, NY 11973-5000

${ }^{2}$ Department of Physics and Astronomy, Rutgers University, Piscataway, NJ 08854

${ }^{3}$ Bell Laboratories, Lucent Technologies, Murray Hill, NJ 07974

\begin{abstract}
We report $\mathrm{x}$-ray scattering studies of polarons and tilt ordering in the hightemperature, paramagnetic insulating phase of $\mathrm{La}_{0.7} \mathrm{Ca}_{0.3} \mathrm{MnO}_{3}$. The temperature dependence of scattering due to individual and correlated polarons was measured, and the latter was found to disappear at $\sim 400 \mathrm{~K}$. The scattering due to tilt ordering, which was observed by tuning the incident photon energy near the $\mathrm{La}_{1}$ edge, was also measured as a function of temperature. The destruction of tilt ordering at $\sim 690 \mathrm{~K}$ is believed to be associated with the orthorhombic-rhombohedral structural transition.
\end{abstract}

\section{INTRODUCTION}

The colossal magnetoresistance (CMR) effect exhibited by some manganese oxides-or manganites-has led to a renewed interest in these complex materials over the past decade [1]. One prototypical CMR manganite is the 3D perovskite of chemical formula $\mathrm{La}_{1-\mathrm{x}} \mathrm{Ca}_{\mathrm{x}} \mathrm{MnO}_{3}$. In the doping range of $0.2<\mathrm{x}<0.5, \mathrm{La}_{1-\mathrm{x}} \mathrm{Ca}_{\mathrm{x}} \mathrm{MnO}_{3}$ is a paramagnetic insulator at room temperature and becomes a ferromagnetic metal - with a concomitant drop in resistivity-at low temperatures. The metallic behavior in the ferromagnetic state has long been ascribed to double exchange between mixed-valence $\mathrm{Mn}$ ions [2]. In this interaction, the hopping of an e electron from a $\mathrm{Mn}^{3+}$ ion to a $\mathrm{Mn}^{4+}$ ion requires ferromagnetic ordering due to a large Hund coupling between the $e_{g}$ electron and the localized $t_{2 g}$ electrons. In the past several years, double exchange has been supplemented with additional ingredients such as electron-phonon coupling [3,4], but a complete description of the CMR effect remains to be formulated.

One recent avenue of investigation into the origin of the CMR effect concerns the role played by phase separation. In this picture, the physics of CMR manganites is dominated by inhomogeneities arising from the coexistence of competing phases [5]. When applied to $\mathrm{La}_{1-\mathrm{x}} \mathrm{Ca}_{\mathrm{x}} \mathrm{MnO}_{3}$, phase separation studies emphasize the importance of understanding not only the ferromagnetic metallic phase, but the paramagnetic insulating phase as well. With this goal in mind, we report $\mathrm{x}$-ray scattering studies of the paramagnetic insulating phase of $\mathrm{La}_{0.7} \mathrm{Ca}_{0.3} \mathrm{MnO}_{3}$.

Two different structural distortions are believed to occur in the paramagnetic insulating phase of $\mathrm{La}_{0.7} \mathrm{Ca}_{0.3} \mathrm{MnO}_{3}$ : one is associated with the formation of polarons, which are caused by strong electron-phonon coupling, and the other arises from tilt ordering of the $\mathrm{MnO}_{6}$ octahedra. Considering first the polarons, evidence of polarons in 
$\mathrm{La}_{1-\mathrm{x}} \mathrm{Ca}_{\mathbf{x}} \mathrm{MnO}_{3}$, for $\mathrm{x} \approx 0.3$, has been obtained using a variety of techniques [6-9], and more recently, interest has turned to studies of polaron-polaron interactions [10-15]. $\mathrm{X}$-ray scattering techniques have proved to be well-suited to such polaron studies since they are directly sensitive to both polarons and their correlations. Specifically, $x$-ray scattering can be used to study diffuse scattering around Bragg peaks due to individual polarons, and resolved peaks due to correlated polarons. In the studies described in this paper, the observation of both types of scattering is reported. The scattering due to correlated polarons is found to disappear at $\sim 400 \mathrm{~K}$. Interestingly, this temperature corresponds closely to the $1.4 \mathrm{~T}_{\mathrm{c}}$ value at which the polaron picture has recently been reported to break down in transport measurements in this material [16].

The other structural distortion that occurs in the paramagnetic insulating phase of $\mathrm{La}_{0.7} \mathrm{Ca}_{0.3} \mathrm{MnO}_{3}$, as mentioned above, arises from tilt ordering of the $\mathrm{MnO}_{6}$ octahedra the so-called $\mathrm{GdFeO}_{3}$-type distortion. Such tilt ordering phenomena are well-known $[17,18]$, but the development of resonant $x$-ray scattering techniques has resulted in a new probe. As described in a recent paper [19], resonant (Templeton) scattering is observed at the tilt order wavevector as a consequence of the two different local environments at the cation site, which are caused by the tilt ordering. As a result, the peak intensity observed at resonance is a measure of tilt order, and the width is inversely related to the tilt order correlation length. In $\mathrm{La}_{0.7} \mathrm{Ca}_{0.3} \mathrm{MnO}_{3}$, we observe such a peak near the $\mathrm{La}_{1}$ absorption edge, and in this paper we report the temperature dependence of its intensity and correlation length.

Taken together, measurements of the two types of structural distortion are an important means of characterizing the paramagnetic insulating phase of $\mathrm{La}_{0.7} \mathrm{Ca}_{0.3} \mathrm{MnO}_{3}$. These measurements also provide an opportunity to investigate possible coupling between tilting of the $\mathrm{MnO}_{6}$ octahedra and the formation of individual and correlated polarons. In what follows, we describe the phase behavior of $\mathrm{La}_{0.7} \mathrm{Ca}_{0.3} \mathrm{MnO}_{3}$, the experimental set-up, and the results of our measurements.

\section{EXPERIMENT}

At low temperatures, $\mathrm{La}_{0.7} \mathrm{Ca}_{0.3} \mathrm{MnO}_{3}$ is a ferromagnetic metal with orthorhombic symmetry Pbnm. It exhibits a metal-insulator transition at $\sim 250 \mathrm{~K}$, above which it is a paramagnetic insulator [20]. At a much higher temperature of $\sim 720 \mathrm{~K}, \mathrm{La}_{0.7} \mathrm{Ca}_{0.3} \mathrm{MnO}_{3}$ goes through a structural transition into a rhombohedral phase [21].

The (110)-oriented single-crystal of $\mathrm{La}_{0.7} \mathrm{Ca}_{0.3} \mathrm{MnO}_{3}$ that was the subject of our investigations was grown using floating zone techniques at Bell Laboratories. The mosaic width, as measured at the (220) Bragg peak (in orthorhombic, Pbnm notation), was $\sim 0.2$ degrees. The $x$-ray scattering measurements were carried out at the National Synchrotron Light Source on bending magnet beamline X22C. This beamline is equipped with a bent, toroidal focusing mirror and a $\mathrm{Ge}(111)$ double-crystal monochromator arranged in a vertical scattering geometry. Two different detector configurations were utilized for the measurements: low- and high-resolution configurations employing $\mathrm{PG}(002)$ and $\mathrm{Ge}(111)$ analyzer crystals, respectively.

In the paramagnetic insulating phase of $\mathrm{La}_{0.7} \mathrm{Ca}_{0.3} \mathrm{MnO}_{3}$, structural distortions associated with the formation of polarons were investigated using the low-resolution 
detector configuration. The incident photon energy was set to $10 \mathrm{keV}$, and reciprocal space scans were carried out as a function of temperature in a closed-cycle displex $(\mathrm{T} \leq 300 \mathrm{~K})$ and a furnace $(\mathrm{T} \geq 300 \mathrm{~K})$. For studies of tilt ordering, resonant $\mathrm{x}$-ray scattering techniques were employed. The incident photon energy was set near the $\mathrm{La} \mathrm{L}_{1}$ absorption edge, and the high-resolution detector configuration was utilized. The sample was placed in a furnace and the temperature dependence of the tilt ordering was measured at the (030) reflection-a forbidden reflection in Pbnm symmetry.

One complication of the furnace measurements is a lack of calibration of the absolute temperature. This is due to the fact that the thermocouple used in the furnace was not attached to the sample, and therefore the temperatures reported for T $>300 \mathrm{~K}$ measurements are believed to be an overestimate of the actual sample temperature.

\section{RESULTS}

Reciprocal space mesh scans around the (440) Bragg peak were carried out at several temperatures, and results at 220,300, and $643 \mathrm{~K}$ are displayed in Figure 1 . The most striking difference between the 220 and $300 \mathrm{~K}$ meshes is the shape of the diffuse scattering. Specifically, the extension of the Bragg peak tails in the [100] and [010] directions, which is also observed at a temperature of $260 \mathrm{~K}$ (not shown), is prominent at the higher temperature. This diffuse scattering can be attributed to the presence of individual, uncorrelated polarons, which form above the metal-insulator transition in the paramagnetic insulating phase.

Above room temperature, the symmetric, four-fold extension of the tails of the (440) Bragg peak is less pronounced, as can be seen in Figure 1(c). It is not clear if this is due to a decrease in the number of individual polarons, a reduction in the magnitude of the structural distortion associated with the polarons, or is caused by an overall increase in thermal diffuse scattering, which swamps the diffuse scattering contribution arising from the individual polarons.

Another noticeable difference in the high-temperature mesh scan is the absence of twin domain peaks. An example is the peak at (4.5 3.5 0) in Figures 1(a) and 1(b), which is actually the (441) reflection of a twin domain. The (441) reflection is an allowed Bragg peak in Pbnm symmetry, but (HHL) reflections are forbidden in the rhombohedral phase for $L$ odd. Therefore the absence of the twin domain peak in Figure 1(c) indicates that the orthorhombic-rhombohedral structural transition occurs at a temperature below $643 \mathrm{~K}$. It should be noted that this upper limit on the structural transition temperature differs from that reported by Uehara, Kim, and Cheong [21], and this discrepancy remains to be reconciled.

In order to study any correlations among the polarons, reciprocal space scans near the (440) Bragg peak were measured as a function of temperature. As reported in an earlier paper [14], broad peaks with ordering wavevectors of $\left(\begin{array}{ll}0.500) & 0\end{array}\right)$ and $(00.50)$ are observed just above the metal-insulator transition temperature. In Figure 2, we show the temperature dependence of one of these peaks for $T \geq 303 \mathrm{~K}$. The peak decreased in intensity with increasing temperature, while the correlation length was found to be independent of temperature, with a magnitude of $\sim 2$ lattice constants. The peak 

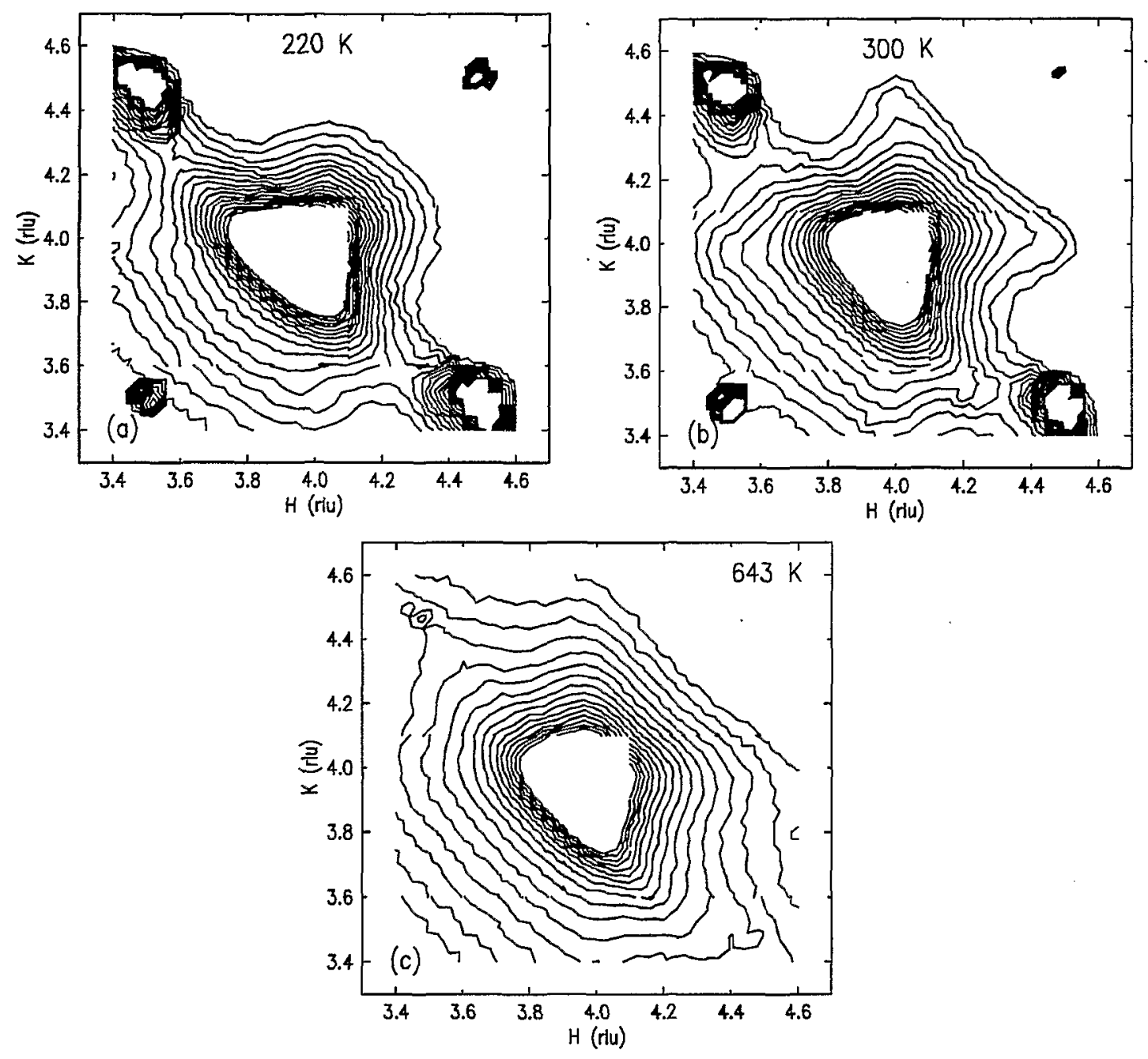

Figure 1. Reciprocal space mesh scans measured at temperatures of 220 (a), 300 (b), and $643 \mathrm{~K}(\mathrm{c})$. The contours are on a logarithmic scale, and were calculated over identical ranges for the three sets of data.

disappeared at a temperature of $\sim 400 \mathrm{~K}$. Interestingly, this corresponds closely to the temperature at which the polaron picture is reported to break down [16], which suggests that the correlated polarons observed in the paramagnetic insulating phase for $T<400 \mathrm{~K}$ may be responsible for the anomalous transport phenomena reported in this temperature regime.

Turning now to the tilt ordering, we have investigated such ordering by studying the forbidden (030) reflection at resonance. The energy dependence of the scattering intensity of this reflection as the incident beam was tuned through the $\mathrm{La}_{1}$ edge is shown in Figure 3. A large resonant peak is observed at $6.275 \mathrm{keV}$, with a full-width of $\sim 10 \mathrm{eV}$. We associate this peak with a $2 \mathrm{~s} \rightarrow 6 \mathrm{p}$ dipole transition, and note that these data are qualitatively similar to those reported for measurements at the $\mathrm{La}$ and $\operatorname{Pr} \mathrm{L}_{1}$ edges for $\mathrm{LaMnO}_{3}$ and $\mathrm{Pr}_{1-\mathrm{x}} \mathrm{Ca}_{\mathrm{x}} \mathrm{MnO}_{3}(\mathrm{x}=0.25,0.4)$, respectively [19]. 


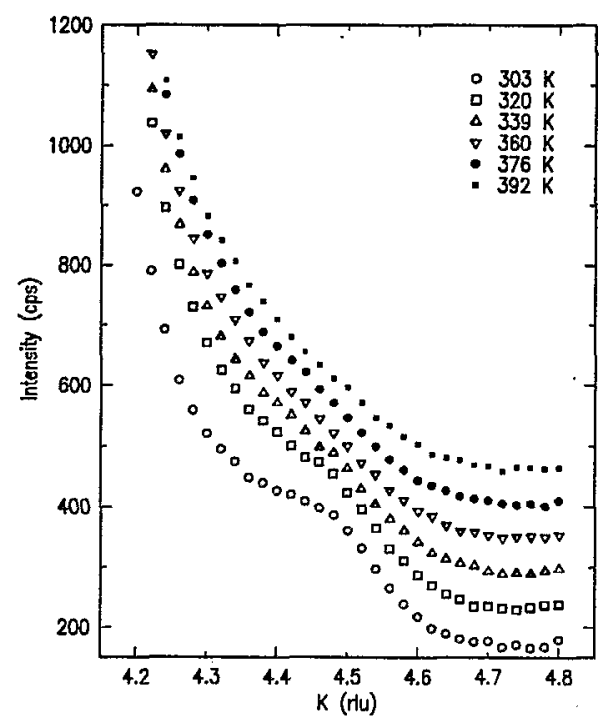

Figure 2. Temperature dependence of the $(44.50)$ peak. Note that data have been shifted for clarity.

The temperature dependence of the tilt ordering was measured from $300 \mathrm{~K}$ until the ordering was observed to disappear at $\sim 690 \mathrm{~K}$. These data were normalized to the (040) Bragg peak intensity in order to eliminate effects of sample motion as a function of temperature. Between room temperature and $690 \mathrm{~K}$, the normalized intensity of the (030) peak decreased with increasing temperature, with no observable broadening. A rapid

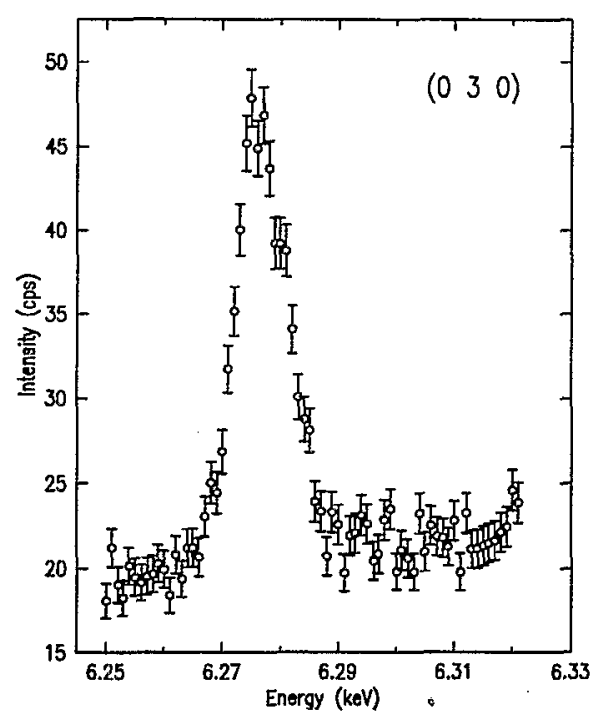

Figure 3. Energy dependence of the scattering intensity at the (030) reflection. 


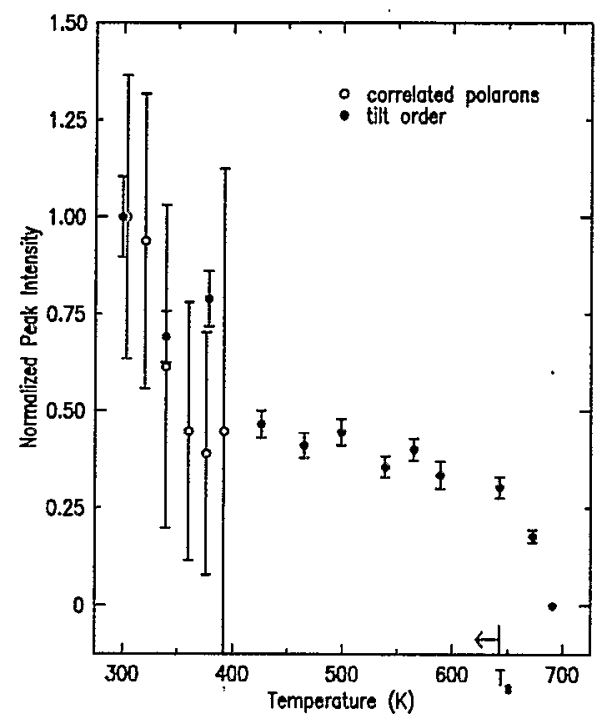

Figure 4. Temperature dependence of peak intensity at (4 4.5 0) (०) and (030) (•), normalized to the (440) and (040) Bragg peaks, respectively. Both sets of data have been scaled to unity at room temperature. The temperature at which the twin domains were no longer observed is indicated by $T_{s}$ (see text).

decrease in intensity above the orthorhombic-rhombohedral structural transition was observed (see Figure 4), which suggests that the destruction of tilt ordering may be associated with this transition. We note that this connection with the high-temperature structural transition is consistent with the phenomenology exhibited by $\mathrm{LaMnO}_{3}$ [19].

In Figure 4, the temperature dependence of the normalized intensities of the peaks due to correlated polarons and tilt ordering are displayed. The temperature at which twin domain peaks are no longer observed is indicated by $T_{s}$, which is therefore an upper limit on the orthorhombic-rhombohedral structural transition temperature. An intriguing question concerns the possibility of a coupling between the correlated polarons and tilt ordering. Unfortunately, the data in Figure 4 are not definitive and further studies are clearly required.

\section{SUMMARY}

We have reported $\mathrm{x}$-ray scattering studies of the structural distortions in the paramagnetic insulating phase of $\mathrm{La}_{0.7} \mathrm{Ca}_{0.3} \mathrm{MnO}_{3}$. Scattering due to individual polarons, correlated polarons, and tilt ordering of the $\mathrm{MnO}_{6}$ octahedra was studied as a function of temperature. Two transitions were observed in these measurements: the peak due to correlated polarons disappeared at $\sim 400 \mathrm{~K}$, and the tilt ordering was destroyed at $\sim 690 \mathrm{~K}$. The transition at $400 \mathrm{~K}$ coincides with a change in transport behavior that has been interpreted in terms of a breakdown in the polaron picture [16]. This result suggests that 
the correlated polarons play an important role in the low-temperature regime of the paramagnetic insulating phase. The high-temperature transition at the tilt order wavevector may be associated with the orthorhombic-rhombohedral structural transition, which is similar to the phenomenology reported in the $\mathrm{x}=0$ member of the $\mathrm{La}_{1-\mathrm{x}} \mathrm{Ca}_{\mathrm{x}} \mathrm{MnO}_{3}$ series [19]. A possible coupling between the correlated polarons and the tilt ordering remains an open question, and will be the focus of future studies.

\section{ACKNOWLEDGMENTS}

The work at Brookhaven, both in the Physics Department and at the NSLS, was supported by the U.S. Department of Energy, Division of Materials Science, under Contract No. DE-AC02-98CH10886. The work at Rutgers University was supported, in part, by the MRSEC program of the NSF, Grant No. DMR-0080008.

\section{REFERENCES}

1. For a review, see Colossal Magnetoresistive Oxides, edited by Y. Tokura (Gordon \& Breach, London, 1999).

2. C. Zener, Phys. Rev. 82, 403 (1951); P.W. Anderson and H. Hasegawa, Phys. Rev. 100, 675 (1955); P.G. deGennes, Phys. Rev. 118, 141 (1960).

3. H. Roder, J. Zang, and A.R. Bishop, Phys. Rev. Lett. 76, 1356 (1996).

4. A.J. Millis, B.I. Shraiman, and R. Mueller, Phys. Rev. Lett. 77, 175 (1996).

5. E. Dagotto, T. Hotta, and A. Moreo, cond-mat/0012117.

6. S.J.L. Billinge, R.G. DiFrancesco, G.H. Kwei, J.J. Neumeier, and J.D. Thompson, Phys. Rev. Lett. 77, 715 (1996).

7. A. Shengalaya, G. Zhao, H. Keller, and K.A. Muller, Phys. Rev. Lett. 77, 5296 (1996).

8. M.F. Hundley and J.J. Neumeier, Phys. Rev. B 55, 11511 (1997).

9. A. Lanzara, N.L. Saini, M. Brunelli, F. Natali, A. Bianconi, P.G. Radaelli, S-W. Cheong, Phys. Rev. Lett. 81, 878 (1998).

10. K.H. Kim, M. Uehara, and S-W. Cheong, Phys. Rev. B 62, R11945 (2000).

11. G.M. Zhao, Y.S. Wang, D.J. Kang, W. Prellier, M. Rajeswari, H. Keller, T. Venkatesan, C.W. Chu, and R.L. Greene, Phys. Rev. B 62, R11949 (2000).

12. P. Dai, J.A. Fernandez-Baca, N. Wakabayashi, E.W. Plummer, Y. Tomioka, and Y. Tokura, Phys. Rev. Lett. 85, 2553 (2000).

13. C.P. Adams, J.W. Lynn, Y.M. Mukovskii, A.A. Arsenov, and D.A. Shulyatev, Phys. Rev. Lett. 85, 3954 (2000).

14. C.S. Nelson, M. v. Zimmermann, Y.J. Kim, J.P. Hill, Doon Gibbs, V. Kiryukhin, T.Y. Koo, S-W. Cheong, D. Casa, B. Keimer, Y. Tomioka, Y. Tokura, T. Gog, and C.T. Venkataraman, cond-mat/0011502.

15. J.M. Zuo and J. Tao, Phys. Rev. B 63, 060407(R) (2001).

16. S.H. Chun, M.B. Salamon, Y. Tomioka, and Y. Tokura, Phys. Rev. B 61, R9225 (2000).

17. V.M. Goldschmidt, Naturwissenschaften 14, 477 (1926). 
18. J.B. Goodenough, Phys. Rev. 100, 555 (1955).

19. M. v. Zimmermann, C.S. Nelson, Y.J. Kim, J.P. Hill, Doon Gibbs, H. Nakao, Y. Wakabayashi, Y. Murakami, Y. Tomioka, Y. Tokura, C-C. Kao, D. Casa, C. Venkataraman, and T. Gog, cond-mat/0007231.

20. P. Schiffer, A.P. Ramirez, W. Bao, and S-W. Cheong, Phys. Rev. Lett. 75, 3336 (1995).

21. M. Uehara, K.H. Kim, and S-W. Cheong, unpublished. 